\title{
Second thrombolysis with recombinant tissue-type plasminogen activator (rtPA) within three days and the use of tirofiban to stop microembolisation before Carotid Endarterectomy (CEA)
}

\author{
Bander Dallol* \\ Heart of England Trust, UK
}

\section{Introduction}

Thrombolysis for acute cerebral infarction is generally not recommended when there is a history of stroke within the last three months. Although there are reports of thrombolysis being given within three months of a cerebral infarction, questioning the evidence behind current guidelines, this is the first report, as far as we are aware, where a standard dose of rtPA was given for the second time within three days. We also discuss the role of tirofiban in stopping microembolisations prior to endarterectomy.

\section{Case report}

An 84 year old patient presented within 85 minutes of developing a dense left arm weakness and moderate left leg weakness, dysarthria, forced eye deviation to the right and severe left sided neglect, (NIHSS 16). CT head scan showed a hyperdense right Middle Cerebral Artery (MCA) M1 thrombosis with no associated acute parenchymal changes (Figure 1). He had a past medical history of coronary bypass surgery after myocardial infarction, stable chronic leukaemia, and Chronic Obstructive Pulmonary Disease (COPD). He was on Aspirin 75 mg, Bisoprolol, Furosamide, Ramipril and inhalers for COPD. He is the main carer for his wife, uses a stick for walking and has a past history of smoking. The patient was thrombolysed at 185 minutes after symptom onset, using a standard $\mathrm{rtPA}$ dose $(0.9 \mathrm{mg} / \mathrm{kg})$. He fully recovered later that day with NIHSS of 0 . Post thrombolysis CT, the next day, showed resolution of the thrombus with no signs of infarction (Figure 2). Carotid Doppler scan showed no flow in the right Internal Carotid Artery (ICA) in keeping with complete occlusion and mild plaque in the left ICA. He was discharged two days later with no focal neurological deficit. Unfortunately, on the day of discharge he was re-admitted with similar symptoms to the first presentation with NIHSS of 15 . CT scan showed right MCA thrombus in the same branch as before with no parenchymal changes (Figure 3 ). The decision was taken with patient's consent to treat with $\mathrm{rtPA}$, at a standard dose $(0.9 \mathrm{mg} / \mathrm{kg}) 110$ minutes after the second episode, 52 hours and 15 minutes after the first rtPA treatment. The patient was made aware of the potential increased risk of haemorrhage with the second rtPA treatment. By the next day he made full neurological recovery. Post thrombolysis CT, again, showed resolution of the MCA thrombus with no signs of infarction (Figure 4). Repeat Carotid Doppler this time showed some flow in the previously occluded right ICA indicating partial re-canalisation. Transcranial Doppler (TCD) of the right MCA was performed and showed two microemboli in 15 minutes. The patient was started on dual antiplatelet therapy (Aspirin $75 \mathrm{mg}$ and Clopidogrel $75 \mathrm{mg}$ ) as per our hospital protocol. The patient had repeat TCD the next day which showed two microemboli in 20 minutes. After discussion with the vascular surgeons he was started on Tirofiban infusion at a rate of $40 \mathrm{ml} / \mathrm{hr}$ for 30 minutes then $10 \mathrm{ml} / \mathrm{hr}$ thereafter. TCD was repeated daily over the next three days and no microemboli were detected over 20 minutes each time. The patient remained on Tirofiban infusion for seven days before having a Carotid Endarterectomy (CEA) under local anaesthesia. After surgery the patient had no focal neurology on examination. He was and remained cognitively intact, and eventually was discharged home six days after the procedure with Modified Rankin Score of zero.

\section{Discussion}

Current guidelines recommend that thrombolysis is contraindicated if there is a history of stroke within the last three months. The rational for this is to allow brain tissue to become gliotic to reduce the risk of bleeding; therefore, it could be safer to repeat rtPA treatment in small or lacunar stroke within three months. In patients with no evidence of parenchymal damage at least on a CT scan, as it was seen in our patient, it could be argued that rtPA could safely be administered. Many cases have been published on repeat thrombolysis within three months of first treatment however non within three days and using a full standard rtPA dose. Furthermore, Glycoprotein IIb/IIIa antagonist tirofiban is well established treatment in patients undergoing coronary intervention. This is owing to its positive effect in reducing platelets rich thrombus formation. Patients with severe carotid artery stenosis are at risk of generating microemboli therefore are at higher risk of developing further ischaemic stroke. In these patients dual antiplatelets could be used to suppress microemboli but in some patients, as in this case, microemboli continue to form despite dual antiplatelets treatment (Aspirin and Clopidogrel). In these patients tirofiban could be used to abolish microemboli and stabilise plaque before undertaking CEA.

Correspondence to: Bander Dallol, Heart of England Trust, UK, E-mail: b.dallol@nhs.net

Received: December 22, 2017; Accepted: January 16, 2018; Published: January 19,2018 
Dallol B (2018) Second thrombolysis with recombinant tissue-type plasminogen activator (rtPA) within three days and the use of tirofiban to stop microembolisation before Carotid Endarterectomy (CEA)

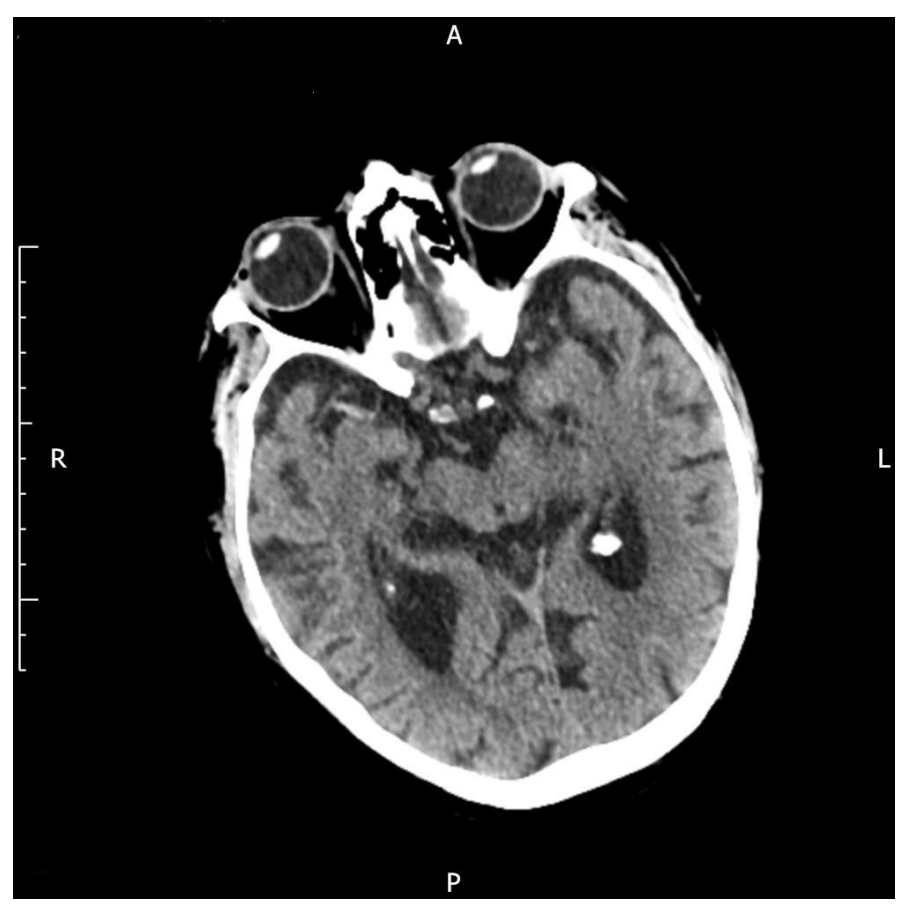

Figure 1. Pre first rtPA showing right MCA thrombus

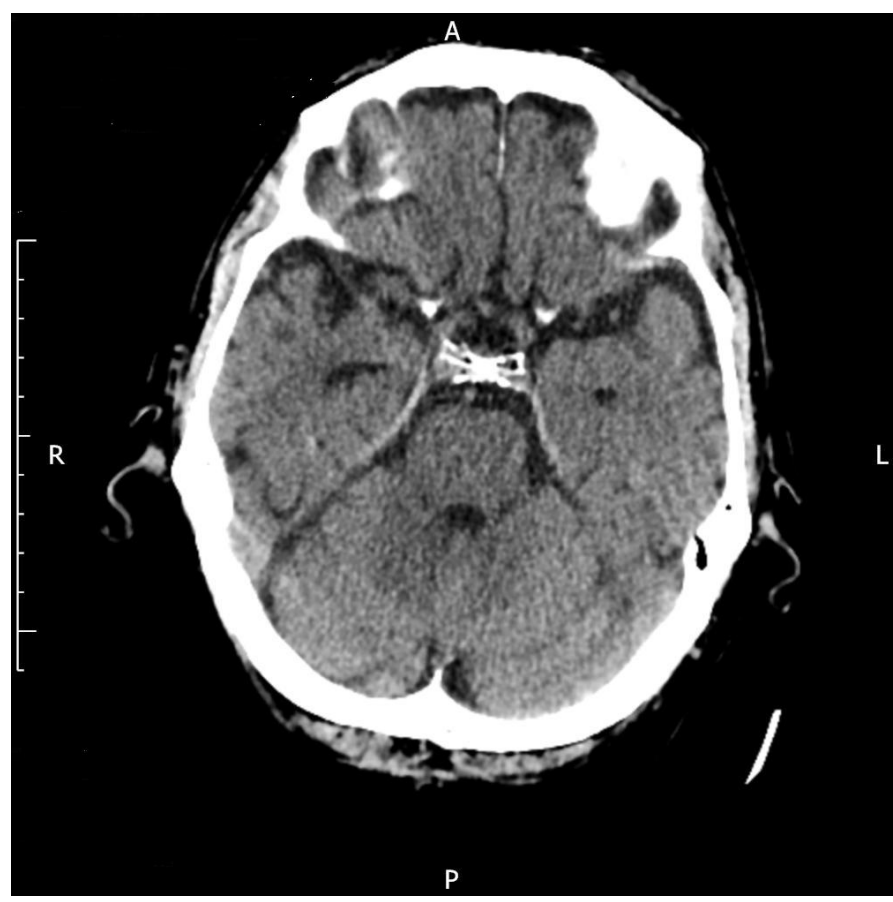

Figure 2. Post first rtPA with no thrombus seen

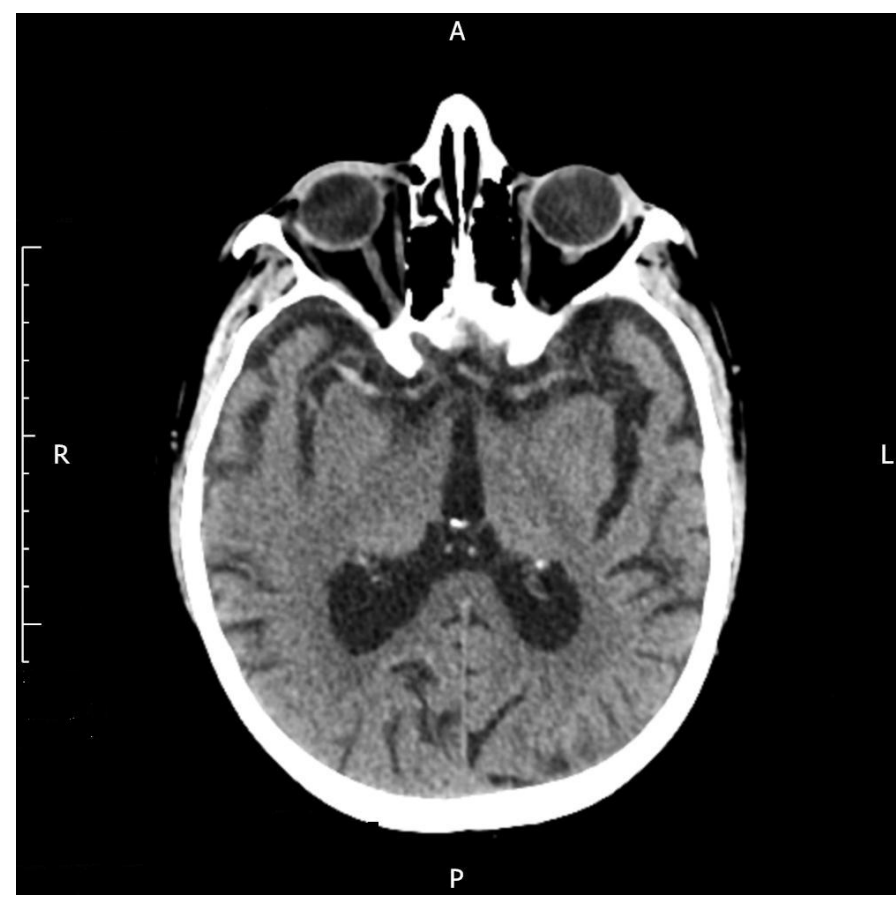

Figure 3. Pre second rtPA showing right MCA thrombus

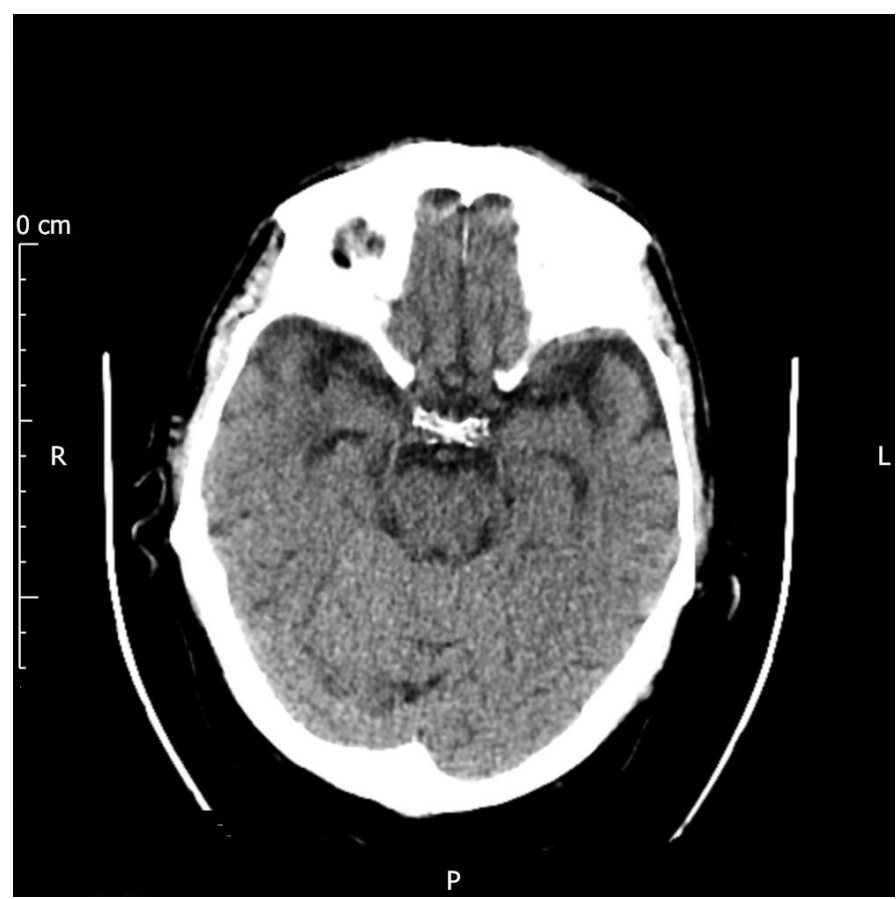

Figure 4. Post second rtPA with no thrombus seen

Copyright: (C2018 Dallol B. This is an open-access article distributed under the terms of the Creative Commons Attribution License, which permits unrestricted use, distribution, and reproduction in any medium, provided the original author and source are credited. 